20

\title{
Многокомпонентный анализ биомаркеров в выдыхаемом воздухе методами диодной лазерной спектроскопии*
}

\author{
(C) Е.В. Степанов, С.Г. Касоев \\ Институт общей фризики им. А.М. Прохорова РАН, \\ 119991 Москва, Россия \\ e-mail: eugenestepanov@yandex.ru
}

Поступила в редакцию 31.01.2019 г.

В окончательной редакции 20.02.2019 г.

Принята к публикации 26.02.2019 г.

\begin{abstract}
Для решения целого ряда задач биомедицинской диагностики предложены методы многокомпонентного спектрального анализа выдыхаемого воздуха, основанные на использовании перестраиваемых диодных лазеров. Показано, что одновременный лазерный спектральный анализ пар $\mathrm{CO}$ и $\mathrm{CO}_{2}, \mathrm{CO}_{2} \mathrm{~N}_{2} \mathrm{O}$ в окрестности $4.7 \mu \mathrm{m}$ может быть использован для исследований газообмена в исследованиях по физиологии дыхания и кардиоваскулярной диагностике, проводимых с применением различных нагрузочных тестов, а также для решения проблем анестезиологии и непрерывного мониторинга вентиляционно-перфузионного отношения. Одновременный анализ $\mathrm{NO}$ и $\mathrm{CO}_{2}$ около $5.4 \mu \mathrm{m}$ может быть полезен для контроля маневра дыхания при исследованиях воспалительных процессов в дистальных отделах легких. Одновременное детектирование $\mathrm{NH}_{3}, \mathrm{CO}_{2}$ и $\mathrm{C}_{2} \mathrm{H}_{4}$ вблизи $10.5 \mu \mathrm{m}$ перспективно для исследований общего обмена и основных метаболических циклов. Одновременный анализ ${ }^{13} \mathrm{CO}_{2}$ и ${ }^{12} \mathrm{CO}_{2}$ в окрестности $2.05 \mu \mathrm{m}$ может быть использован для измерения отношения ${ }^{13} \mathrm{CO}_{2} /{ }^{12} \mathrm{CO}_{2}$ при проведении изотопических дыхательных тестов. Приведены результаты анализа перспективных спектральных диапазонов и взаимного расположения аналитических линий в них. Экспериментально продемонстрирована возможность одновременного детектирования нескольких исследуемых молекул в предложенных спектральных областях, получены лазерные спектры пропускания. Продемонстрирована возможность применения предложенного подхода для анализа микросостава выдыхаемого воздуха.
\end{abstract}

DOI: $10.21883 /$ OS.2019.06.47777.57-19

\section{1. Введение}

Как известно, выдыхаемый воздух представляет собой сложную газовую смесь, содержащую помимо атмосферных газов продукты основного обмена $\left(\mathrm{CO}_{2}, \mathrm{H}_{2} \mathrm{O}\right)$, а также множество газообразных молекул в следовых количествах, некоторые из которых можно использовать в качестве биомаркеров [1-5]. Список таких молекулбиомаркеров достаточно велик. Среди них легкие молекулы типа $\mathrm{CO}, \mathrm{NO}, \mathrm{NO}_{2}, \mathrm{~N}_{2} \mathrm{O}, \mathrm{NH}_{3}, \mathrm{H}_{2} \mathrm{O}_{2}, \mathrm{C}_{2} \mathrm{H}_{4}, \mathrm{C}_{2} \mathrm{H}_{6}$, $\mathrm{CH}_{2} \mathrm{O}, \mathrm{CH}_{4}, \mathrm{CH}_{3} \mathrm{OH}, \mathrm{C}_{2} \mathrm{H}_{5} \mathrm{OH}, \mathrm{CS}_{2}, \mathrm{H}_{2} \mathrm{~S}, \mathrm{C}_{5} \mathrm{H}_{12}, \mathrm{C}_{2} \mathrm{H}_{6}$, $\mathrm{CH}_{2} \mathrm{OHS}$ и другие, концентрации которых в выдохе находятся в диапазоне от $1 \mathrm{ppm}$ до $0.1 \mathrm{ppb}$. Кроме того, для биомедицинской диагностики актуальна высокоточная регистрация относительного содержания изотопических модификаций молекул-метаболитов, обогащенных более редкими изотопами $\left(\mathrm{D},{ }^{13} \mathrm{C},{ }^{18} \mathrm{O},{ }^{15} \mathrm{~N}\right.$ и $\left.{ }^{35} \mathrm{~S}\right)$.

Для исследований микросостава выдыхаемого воздуха актуальны разработка и применение многокомпонентных аналитических систем [6-8]. Одновременный анализ содержания нескольких газообразных веществ позволяет наблюдать корреляции в их выделении с выдыхаемым

\footnotetext{
* The 22nd Annual Conference Saratov Fall Meeting 2018 (SFM'18): VI International Symposium „Optics and Biophotonics“ and XXII International School for Junior Scientists and Students on Optics, Laser Physics \& Biophotonics, September 24-29, 2018, Saratov, Russia. https://www.sgu.ru/structure/fiz/saratov-fall-meeting/previousconferences/saratov-fall-meeting-2018
}

воздухом и, таким образом, исследовать взаимосвязи между различными физиологическими и биохимическими процессами в организме. Следует ожидать, что диагностика, опирающаяся на многокомпонентный газовый анализ, может обладать большей достоверностью, универсальностью, позволит проводить комплексные исследования организма и расширить круг решаемых медикобиологических проблем.

Методы диодной лазерной спектроскопии (ДЛС) предоставляют достаточно широкие возможности для реализации многокомпонентного газового анализа состава выдыхаемого воздуха [9-16]. При использовании перестраиваемых диодных лазеров (ПДЛ) высокочувствительный анализ многих молекулярных газов может быть осуществлен в рамках единого физического подхода и единого аналитического спектрального устройства. Наиболее существенным для реализации этого подхода является широкий спектральный диапазон, перекрываемый всем семейством ПДЛ (от видимого до среднего ИК), что позволяет настроиться на удобные для анализа линии поглощения исследуемых газообразных молекул. Низкий уровень амплитудных и частотных шумов ПДЛ позволяет регистрировать резонансное молекулярное поглощение с чувствительностью к изменению оптической плотности вплоть до $10^{-7}$ и спектральным разрешением $\sim 3 \cdot 10^{-4} \mathrm{~cm}^{-1}$. Использование методов ДЛС для измерения параметров линий поглощения, 
принадлежащих колебательно-вращательным спектрам молекул, как известно $[9,11,13,15,16]$, позволяет реализовать следующие точностные характеристики: точность определения центров линий поглощения - вплоть до $10^{-5} \mathrm{~cm}^{-1}$, погрешность определения величины резонансного поглощения $\sim 0.1 \%$, концентрационная чувствительность в диапазоне от десятков ppt до единиц ppm (в зависимости от типа молекул и используемого спектрально диапазона). Кроме того, важны легкость электронного управления параметрами излучения, в частности быстрой перестройкой частоты, возможность достаточно легко автоматизировать системы на основе таких лазеров и их миниатюрность.

При использовании методов ДЛС для многокомпонентного газового анализа возможно использование нескольких различных подходов, базирующихся, например, на случайном близком расположении аналитических линий; специальных методах накачки ПДЛ, применении нескольких лазеров в одном устройстве и их интеграции с элементами ИК волоконной оптики. В настоящей работе описаны результаты исследований, полученных только при использовании первых двух подходов, поскольку решение задачи мультиплексирования нескольких спектральных каналов требует совершенно иных технических решений.

Цель данных исследований состояла в анализе спектров поглощения ряда молекул-биомаркеров на основании баз спектральных данных высокого разрешения, выборе спектральных участков, наиболее благоприятных для реализации многокомпонентного анализа с помощью одного ПДЛ, и инструментальной реализации и апробации предлагаемого подхода. При этом предложенные методы многокомпонентного лазерного спектрального анализа состава выдыхаемого воздуха были нацелены на решение актуальных задач функциональной диагностики и неинвазивной диагностики заболеваний.

\section{2. Экспериментальные методы}

Исследования базировались на использовании спектрофотометра на основе ПДЛ ближнего и среднего ИК диапазонов, оптическая схема которого показана на рис. 1 [10]. Для поддержания рабочей температуры, которая зависит от типа используемого излучателя, лазеры помещаются в криостат. Необходимая для регистрации молекулярных спектров поглощения длина волны оптического излучения обеспечивается как за счет подбора рабочей температуры лазера, так и за счет вариации амплитуды тока накачки. Для сканирования частоты и регистрации спектров пропускания используется импульснопериодический режим накачки с амплитудой импульсов тока $\sim 0.1-2.0 \mathrm{~A}$, длительностью от 100 до $10^{4} \mu \mathrm{s}$ и частотой повторения $\sim 100 \mathrm{~Hz}$. Перестройка частоты генерации лазера происходит в течение каждого импульса тока накачки со скоростями порядка $10^{2}-10^{5} \mathrm{~cm}^{-1} \mathrm{~s}^{-1}$ (в зависимости от типа ПДЛ) и воспроизводится с высокой точностью от импульса к импульсу. Мощность

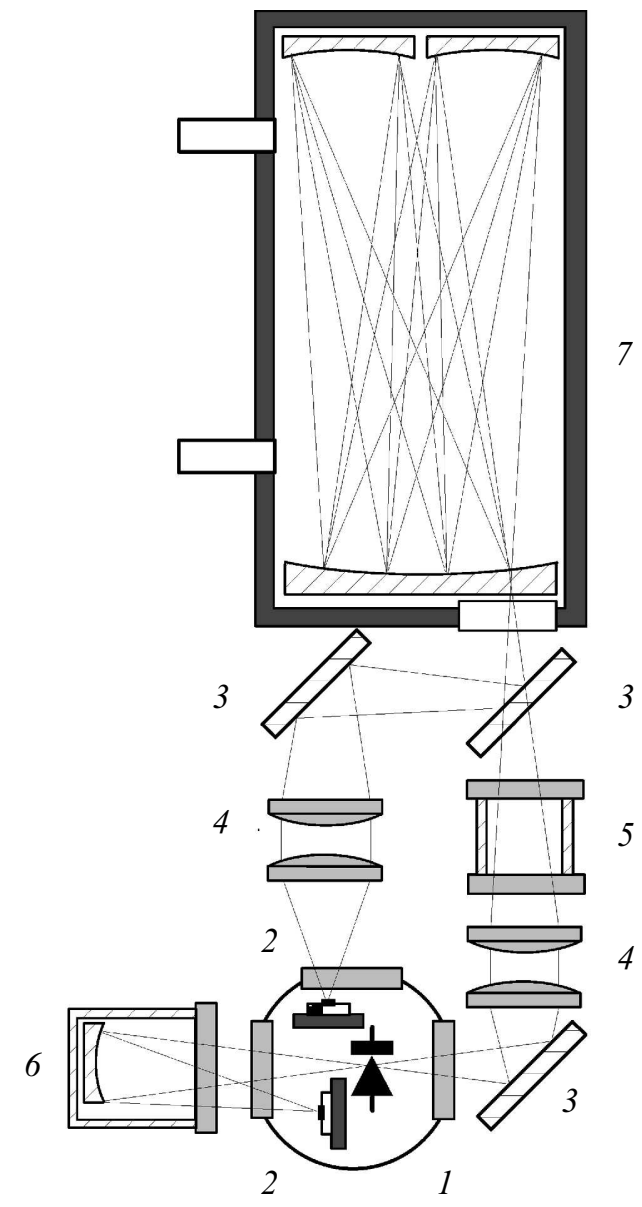

Pис. 1. Оптическая схема спектрофотометра на основе перестраиваемых диодных лазеров. 1 - ПДЛ в криостате, $2-$ ИК фотоприемники в криостате, 3 - поворотные зеркала, 4 двулинзовые ИК объективы, 5 - калибровочная кювета, 6 реперная кювета, 7 - многоходовая аналитическая кювета.

лазерного излучения составляет $0.3-0.5 \mathrm{~mW}$. Выходящее из криостата излучение ПДЛ с помощью линзового объектива из $\mathrm{BaF}_{2}$ вводится в многоходовую кювету (МХК), съюстированную по схеме Вайта. Объем кюветы составляет $\sim 1.21$ при базовой длине кюветы $\sim 30 \mathrm{~cm}$. Оптический путь в МХК составляет $\sim 20 \mathrm{~m}$. После прохождения кюветы лазерное излучение фокусируется на охлаждаемый фотодиод $\mathrm{InSb}$, обладающий быстродействием $\sim 20$ ns. Для исследования спектров поглощения выдыхаемого воздуха последний напускается в многоходовую кювету.

Для управления спектрофотометром и регистрации лазерных спектров пропускания используется разработанный нами специализированный программно-аппаратный комплекс. В нем блок управления и регистрации спектров построен с использованием нескольких программируемых логических матриц (FPGA Cyclone 2, Altera) и быстродействующего, $5 \mathrm{MHz}, 16-$-ти разрядного сигма-дельта АЦП, 10MSPS (Texas Instruments). Комплекс имеет два независимых канала сбора и обработки спектральной информации и связь с персональным 
компьютером через скоростной порт USB 2.0 (скорость обмена данными - $480 \mathrm{Mbit} / \mathrm{s}$ ). Программное обеспечение, поддерживающее работу комплекса, работает в среде WinXP/WinVista и позволяет управлять работой спектрального устройства с помощью персонального компьютера, а также проводить накопление, обработку, визуализацию и хранение регистрируемых данных.

Параметры программно-аппаратного комплекса позволяют регистрировать лазерные спектры со следующими характеристиками: длительность лазерного импульса (развертки регистрируемого спектра) от 100 до $30000 \mu \mathrm{s}$, частота повторения от $10 \mathrm{~Hz}$ до $10 \mathrm{kHz}$, протяженность спектра $2-15 \mathrm{~cm}^{-1}$, скорость перестройки оптической частоты $10^{2}-10^{4} \mathrm{~cm}^{-1} / \mathrm{s}$. При этом обеспечивается регистрация от 500 до 150000 точек на спектр и от 30 до 5000 точек на одну аналитическую линию поглощения. За счет использования упомянутого выше АЦП, позволяющего проводить оцифровку формы лазерных спектров с временным разрешением до $200 \mathrm{~ns}$, скорость регистрации лазерных спектров составляет от $100 \mu \mathrm{s}$ (одиночный короткий лазерный импульс без накопления) до нескольких минут (суммирование более 10000 импульсов длительностью $4 \mathrm{~ms}, 20000$ точек на спектр, при частоте повторения $100 \mathrm{~Hz})$. При этом высокая скорость оцифровки сигнала и обмена данными между системой регистрации и управляющим компьютером позволяет регистрировать без пропусков все лазерные импульсы в двух регистрирующих каналах одновременно. Регистрация формы лазерных импульсов с такими характеристиками позволяет осуществить качественную запись контуров линий поглощения, детектируемых с помощью ПДЛ, и их производных по времени, что важно для достижения требуемых характеристик спектрального анализа по точности, чувствительности, селективности и быстродействию. Использование высокоскоростного протокола USB 2.0 для передачи данных в компьютер позволяет избежать потери полезной информации и увеличить быстродействие системы.

\section{3. Результаты и обсуждение}

Для многокомпонентного анализа газообразных молекул-биомаркеров нами были апробированы два различных подхода, которые базируются на случайном близком расположении аналитических линий и специальных методах накачки ПДЛ.

\section{1. Использование случайного близкого расположения аналитических линий детектируемых молекул}

Ряд задач многокомпонентного анализа может быть решен благодаря интерференции колебательно-вращательных (КВ) полос поглощения исследуемых газообразных соединений. В этом случае линии поглощения различных молекул могут оказаться в пределах одной моды

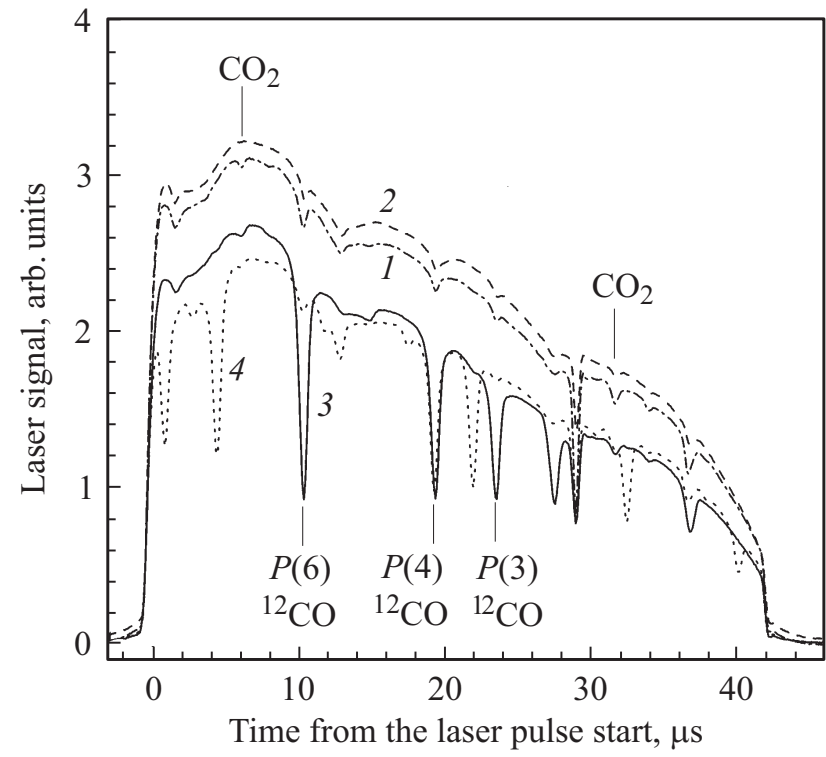

Рис. 2. Лазерный спектр пропускания выдыхаемого воздуха (1), атмосферного воздуха (2), калибровочной смеси $\mathrm{CO}: \mathrm{N}_{2}(3)$ и обогащенной по ${ }^{13} \mathrm{C}^{16} \mathrm{O}$ и ${ }^{12} \mathrm{C}^{18} \mathrm{O}$ изотопической смеси с азотом (4) при атмосферном давлении. Длина оптического пути в МХК - $18.6 \mathrm{~m}$, в калибровочных кюветах $5 \mathrm{~cm}$.

ПДЛ или в соседних модах и могут быть зарегистрированы практически одновременно. При этом существенно, чтобы КВ спектры детектируемых газообразных соединений были достаточно разрежены, т.е. существовали микроокна прозрачности. В этом случае, используя ПДЛ в качестве источника, можно рассчитывать на одновременное детектирование $2-3$ различных молекул с помощью одного лазера. Выбор комбинации близкорасположенных линий поглощения, подходящих для решения той или иной аналитической задачи, требует предварительного моделирования КВ спектров высокого разрешения исследуемых молекул с использованием баз спектральных данных типа HITRAN-2004 [17]. При этом необходим тщательный анализ взаимного расположения и интенсивности линий детектируемых молекул и линий поглощения мешающих газов, в первую очередь воды или углекислого газа.

\section{Одновременное детектирование $\mathrm{CO}$ и $\mathrm{CO}_{2}$ для исследований кислород-транспортных свойств гемоглобина при изменяющемся уровне $\mathrm{CO}_{2}$ в крови}

Одновременное детектирование $\mathrm{CO}$ и $\mathrm{CO}_{2}$ в выдыхаемом воздухе было реализовано нами с помощью ПДЛ диффузионного типа, генерирующего вблизи $2110 \mathrm{~cm}^{-1}$. В данном спектральном диапазоне КВ спектры этих линейных молекул состоят из отдельных достаточно регулярно расположенных линий поглощения, что облегчает выбор подходящей аналитической пары линий и режима генерации ПДЛ. Для высокочувствительного лазерного 
анализа СО были использованы линии поглощения фундаментальной колебательно-вращательной полосы 1-0, расположенной вблизи $4.7 \mu \mathrm{m}$ (рис. 2). В силу линейности молекулы моноокиси углерода эта полоса является единственной полосой фундаментального поглощения СО и состоит из отдельных изолированных линий поглощения, удаленных друг от друга на $\sim 4 \mathrm{~cm}^{-1}$. Наиболее интенсивные линии $P$ - и $R$-ветвей со значениями $J$ от 5 до 10 расположены соответственно в частотных интервалах $2100-2125 \mathrm{~cm}^{-1}$ и $2160-2180 \mathrm{~cm}^{-1}$. Их интенсивности составляют $3-4 \cdot 10^{-19} \mathrm{~cm}^{-1} / \mathrm{mol} \cdot \mathrm{cm}^{-2}$, а коэффициент столкновительного уширения воздухом $0.06-0.08 \mathrm{~cm}^{-1} \mathrm{~atm}^{-1}$ [17]. Полоса $1-0$ СО интерферирует с несколькими слабыми полосами $\mathrm{CO}_{2}$ и $\mathrm{H}_{2} \mathrm{O}$. В данном случае эта интерференция не является фактором, ухудшающим условия детектирования СО в выдыхаемом воздухе, поскольку спектры достаточно разрежены. В $P$-ветви наименее чувствительны к $\mathrm{CO}_{2}$ и $\mathrm{H}_{2} \mathrm{O}$ линии $P(3), P(4), P(5)$ и $P(10)$. $P$-ветвь наиболее привлекательна с точки зрения одновременного детектирования в выдыхаемом воздухе $\mathrm{CO}$ и $\mathrm{CO}_{2}$, что актуально для исследований в области физиологии дыхания.

Линии $\mathrm{CO}_{2}$, принадлежащие горячей полосе 20001-01101, имеют интенсивность поглощения на $\sim 4$ порядка более низкую, чем линии $P$-ветви фундаментальной полосы 1-0 СО. При концентрации СОв выдыхаемом воздухе на уровне 1 ppm и $\mathrm{CO}_{2}$ на уровне $3 \%$ такая разница в интенсивностях дает близкое по величине поглощение для обоих газов и позволяет производить измерение концентраций в одном динамическом диапазоне регистрирующей аппаратуры.

В силу достаточно регулярного расположения линий $\mathrm{CO}$ по спектру, актуальной является точная идентификация используемых для анализа линии поглощения. Простой способ решения этой проблемы связан с использованием изотопомеров моноокиси углерода, т. е. молекул ${ }^{13} \mathrm{C}^{16} \mathrm{O}$ и ${ }^{12} \mathrm{C}^{18} \mathrm{O}$. Благодаря изотопическому смещению центров полос ${ }^{12} \mathrm{C}^{16} \mathrm{O},{ }^{13} \mathrm{C}^{16} \mathrm{O}$ и ${ }^{12} \mathrm{C}^{18} \mathrm{O}$ и небольшой разнице их вращательных постоянных спектры этих молекул в совокупности образуют достаточно специфичную и неповторяющуюся картину (рис. 2), которую удобно использовать для частотной привязки спектра и идентификации линий. Грубое предварительное определение рабочего спектрального диапазона каждого ПДЛ, с точностью до нескольких $\mathrm{cm}^{-1}$, проводится с помощью монохроматора или берется из технического паспорта лазера. При проведении точной частотной привязки могут поочередно записываться спектры пропускания изотопических модификаций $\mathrm{CO}, \mathrm{CO}_{2}$ и паров воды. Далее взаимное расположение линий поглощения и их интенсивности сравниваются с табличными данными, например, базы молекулярных спектров высокого разрешения HITRAN-2004 [17], и проводится идентификация линий.

Одновременное детектирование $\mathrm{CO}$ и $\mathrm{CO}_{2}$ с помощью одного ПДЛ было использовано нами при изучении

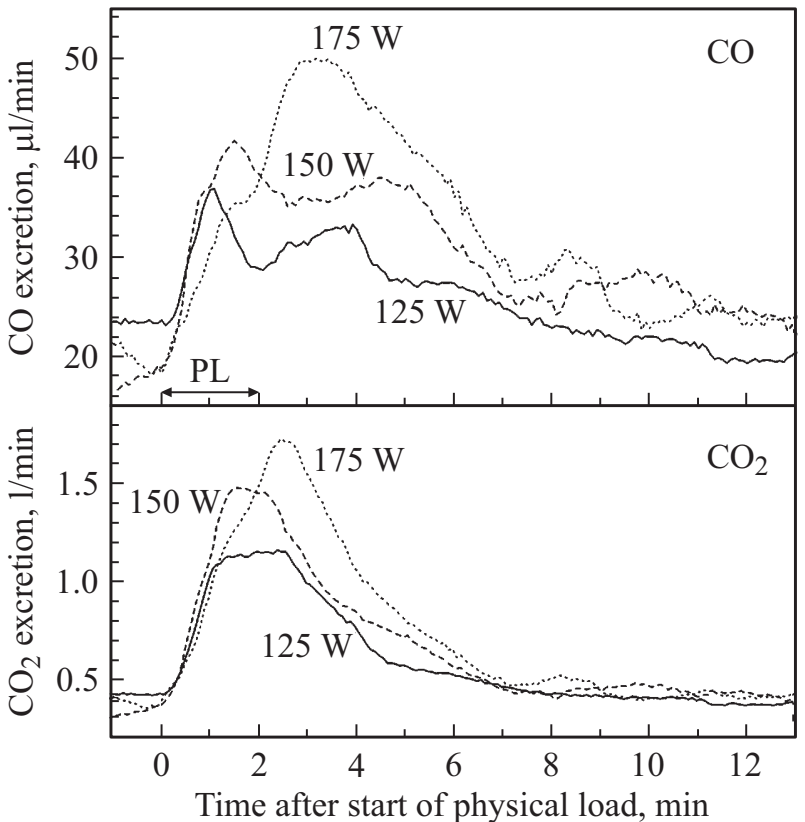

Рис. 3. Динамика выделения $\mathrm{CO}$ и $\mathrm{CO}_{2}$ с выдыхаемым воздухом, обусловленная физической нагрузкой (PL) длительностью 2 min различной мощности 125,150 и $175 \mathrm{~W}$.

закономерностей выделения этих газов при дозированной физической нагрузке, при проведении гиперкапнических тестов для мышей, а также в исследованиях зависимости концентрации СО в выдыхаемом воздухе от времени задержки. В этих исследованиях данные о концентрации $\mathrm{CO}_{2}$ применялись для выявления зависимости эффективности связывания СО гемоглобином крови от концентрации растворенного в крови $\mathrm{CO}_{2}$ и учета факторов, обусловленных накоплением и/или повышением концентрации $\mathrm{CO}_{2}$ в крови вследствие используемых тестовых нагрузок. На рис. 3 показана динамика выделения $\mathrm{CO}$ и $\mathrm{CO}_{2}$ с выдыхаемым воздухом испытуемого, обусловленная физической нагрузкой длительностью $2 \mathrm{~min}$ различной мощности: 125,150 и $175 \mathrm{~W}$. Выделение $\mathrm{CO}$ и $\mathrm{CO}_{2}$ определялось на основании измерения концентрации этих газов в выдыхаемом воздухе с помощью лазерного анализатора и объема выдыхаемого воздуха в единицу времени.

\section{Одновременное детектирование $\mathrm{CO}$ и $\mathrm{N}_{2} \mathrm{O}$ для исследований зависимости газотранспортных свойств легочной мембраны от интенсивности кровотока}

Уникальные диагностические возможности предоставляет также интерференция КВ полос 1-0 $\mathrm{CO}$ и $0001-0000 \mathrm{~N}_{2} \mathrm{O}$, расположенных вблизи $4.6 \mu \mathrm{m}$, благодаря которой можно одновременно измерять концентрации этих двух газов в выдыхаемом воздухе с использованием одного ПДЛ. 
При добавлении во вдыхаемый воздух небольших концентраций закиси азота $\left(\mathrm{N}_{2} \mathrm{O}\right)$ она эффективно диффундирует через легочную мембрану и хорошо растворяется в липидах клеток крови [18]. Скорость изменения ее концентрации в выдыхаемом воздухе за один дыхательный цикл может служить мерой интегральной перфузии (наполнения кровью) легочных тканей, а при непрерывном контроле с помощью газового анализатора эта величина может быть использована для непрерывного мониторинга кровенаполнения легких. В то же время одновременный анализ выделения эндогенного СО с выдохом позволяет судить об эффективности кислород-транспортных свойств крови и вентиляции легких. Таким образом, при одновременном контроле обоих газов становится возможным непрерывный мониторинг вентиляционно-перфузионного отношения [18], которое является важной характеристикой дыхания организма во многих кардио-респираторных исследованиях с применением разнообразных физиологических тестов.

Для одновременного измерения $\mathrm{CO}$ и $\mathrm{N}_{2} \mathrm{O}$ с помощью одного ПДЛ нами был использован диффузионный лазер, генерирующий в спектральном диапазоне $2160-2180 \mathrm{~cm}^{-1}$, где расположены наиболее подходящие для анализа линии поглощения обоих веществ. Для регистрации СО использовалась одна из наиболее интенсивных линий перехода $1-0, R(5)$, с частотой центра согласно базе данных HITRAN-2004 $2165.601 \mathrm{~cm}^{-1}$, а для регистрации $\mathrm{N}_{2} \mathrm{O}$ - линии $P$-ветви перехода 0001-0000 с большими значениями вращательного момента. Экспериментально измеренные лазерные спектры пропускания представлены на рис. 4. Измеряемые концентрации $\mathrm{CO}$ составляли от 0.5 до $2.5 \mathrm{ppm}$. Концентрация $\mathrm{N}_{2} \mathrm{O}$ во вдыхаемом воздухе поддерживалась около $10 \mathrm{ppm}$, в выдыхаемом воздухе она составляла от 5 до 7 ppm.

Предложенный метод одновременного детектирования $\mathrm{CO}$ и $\mathrm{N}_{2} \mathrm{O}$ в выдыхаемом воздухе был опробован нами в исследованиях микрогазообмена при дозированной физической нагрузке, во время которой существенно меняется как вентиляция легких, так и скорость кровотока.

\section{Одновременное детектирование $\mathrm{NO}$ и $\mathrm{CO}_{2}$ для контроля маневра дыхания при исследованиях воспалительных процессов в дистальных отделах легких}

При измерении концентрации NO в выдыхаемом воздухе с целью использования этой молекулы в качестве маркера воспалительных процессов в верхних дыхательных путях желательно одновременно детектировать и содержание $\mathrm{CO}_{2}$. Это позволяет контролировать правильность проведения дыхательного маневра, применяемого при тестировании, и выбирать индивидуально для каждого испытуемого момент определения бронхиальной составляющей в порции выдыхаемого воздуха.

Одновременная регистрация $\mathrm{NO}$ и $\mathrm{CO}_{2}$ с помощью одного ПДЛ возможна благодаря перекрытию полос
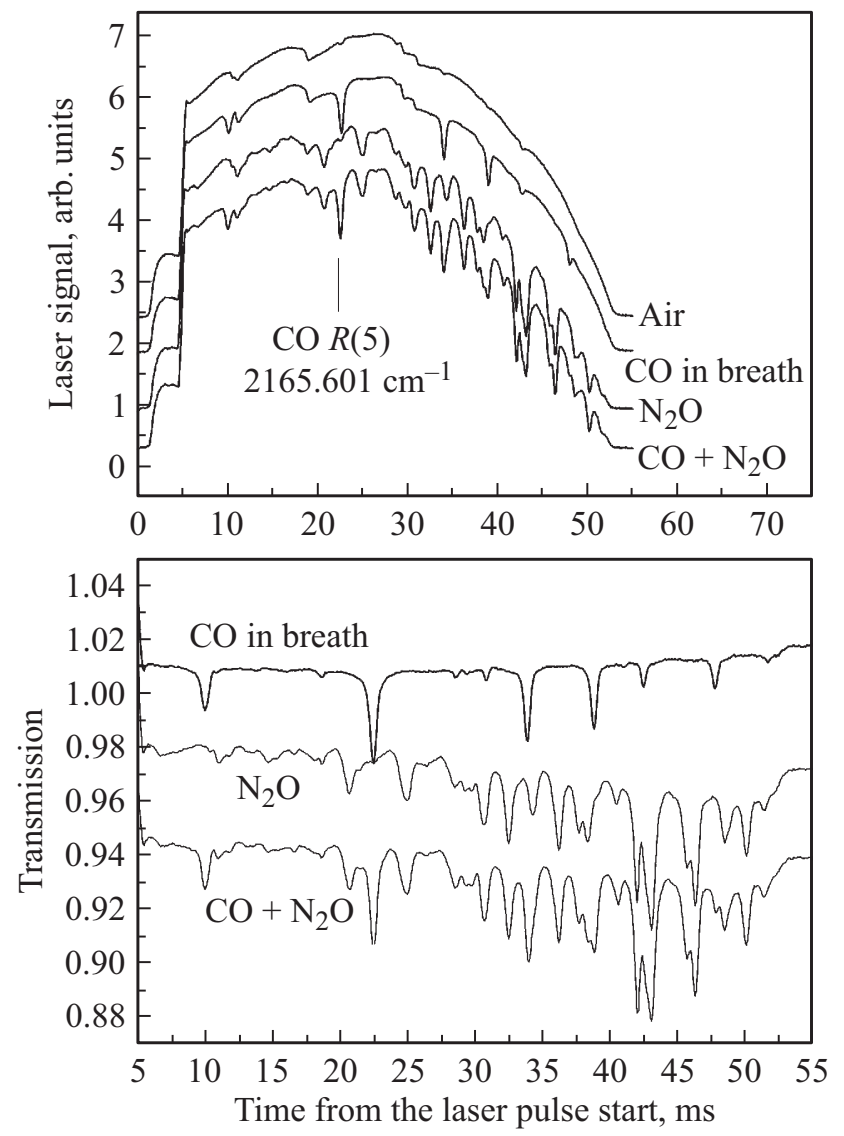

Рис. 4. Лазерные спектры и спектры пропускания выдыхаемого воздуха в спектральном диапазоне, использованном для одновременной регистрации $\mathrm{CO}$ и $\mathrm{N}_{2} \mathrm{O}$. Спектры пропускания на нижнем рисунке смещены по вертикали относительно друг друга во избежание их наложения.

поглощения 1-0 NO и 20003-01101 $\mathrm{CO}_{2}$, имеющему место вблизи $5.5 \mu \mathrm{m}$. Выбор наиболее подходящей аналитической пары линий $\mathrm{NO}$ и $\mathrm{CO}_{2}$ очень ограничен изза сильной интерференции с полосой поглощения воды. Для регистрации NO наиболее подходит дублет $P(7.5)$ с суммарной интенсивностью $\sim 10^{-19} \mathrm{~cm}^{-1} / \mathrm{mol} \cdot \mathrm{cm}^{-2}$, расположенный вблизи $1850.17 \mathrm{~cm}^{-1}$ (рис. 5). Ближайшие пригодные для анализа линии поглощения $\mathrm{CO}_{2} P(41)$ и $P(39)$ полосы 20003-01101 отстоят от дублета NO не более, чем на $1 \mathrm{~cm}^{-1}$, и обладают интенсивностью $\sim 3.6 \cdot 10^{-25} \mathrm{~cm}^{-1} / \mathrm{mol} \cdot \mathrm{cm}^{-2}$. Превышающая 5 порядков разница в интенсивности поглощения аналитических линий позволяет измерять концентрации обеих молекул, составляющих в выдыхаемом воздухе от 5 до $100 \mathrm{ppb}$ для $\mathrm{NO}$ и $3 \%$ для $\mathrm{CO}_{2}$, в едином динамическом диапазоне тракта регистрации. Кроме того, за счет близких коэффициентов экстинкции и достаточного спектрального разделения аналитических линий $\mathrm{NO}$ и $\mathrm{CO}_{2}$ обеспечивается минимальность их взаимного воздействия друг на друга.

Возможность одновременного детектирования NO и $\mathrm{CO}_{2}$ в выдыхаемом воздухе с помощью единого ПДЛ 


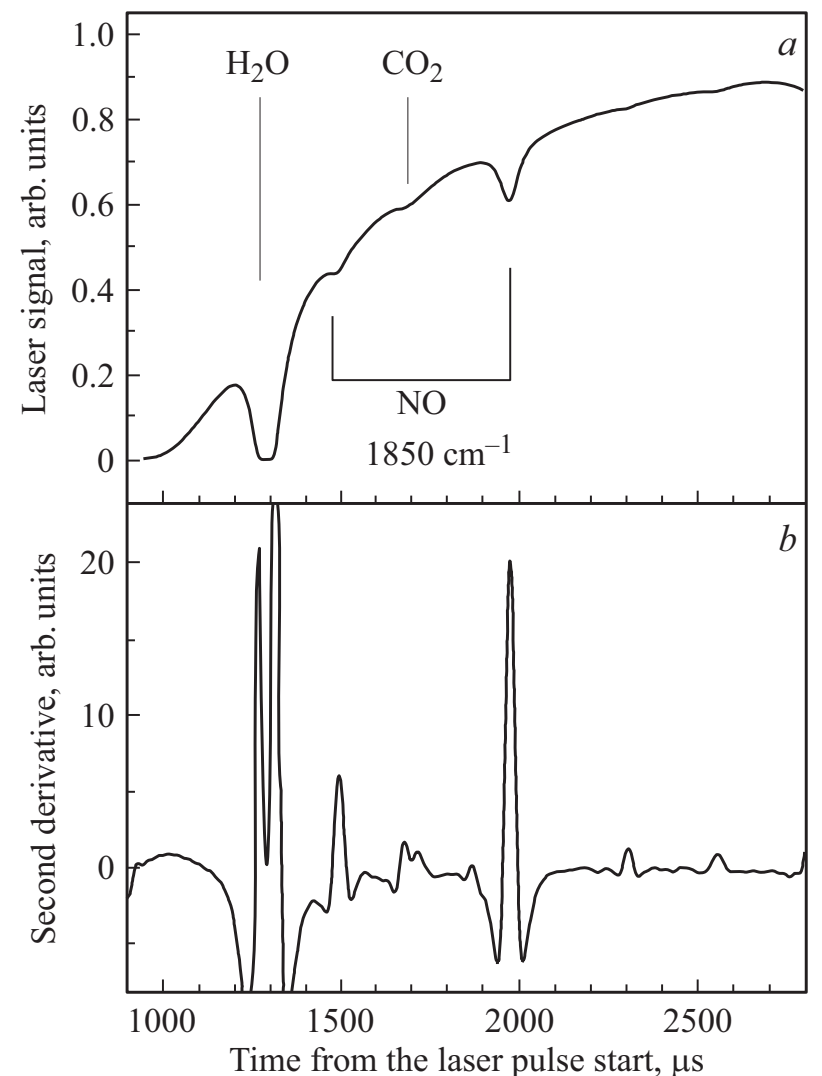

Рис. 5. Лазерный спектр пропускания NO в выдыхаемом воздухе $(a)$ и его вторая производная $(b)$, регистрируемые с помощью ПДЛ в районе $1850 \mathrm{~cm}^{-1}$ при содержании $\mathrm{NO}$ $\sim 1 \mathrm{ppm}$.

была экспериментально продемонстрирована с помощью лазера с двойной гетероструктурой (ДГС) на основе соединения $\mathrm{PbEuSe}$. Лазерный спектр пропускания NO и $\mathrm{CO}_{2}$ вблизи $1850 \mathrm{~cm}^{-1}$ и его вторая производная показаны на рис. 5. Значение концентрации $\mathrm{CO}_{2}$ в анализируемой пробе воздуха использовалось для оперативного контроля дыхательного маневра и выбора момента измерения концентрации NO.

\section{Одновременное детектирование $\mathrm{NH}_{3}$ и $\mathrm{CO}_{2}$ для исследований общего обмена и основных метаболических циклов}

Для исследования закономерностей выделения аммиака организмом информативным является одновременное детектирование $\mathrm{NH}_{3}$ и $\mathrm{CO}_{2}$. Это обусловлено тем, что метаболизм аммиака в организме тесно связан с энергетическим обменом, кислотно-основным состоянием и катаболизмом белков, т.е. с процессами, в которых активно задействована $\mathrm{CO}_{2}$. Одновременный анализ выделения этих двух веществ позволяет наблюдать взаимосвязи между механизмами их образования и утилизации в организме.
В силу сложности и нерегулярности расположения линий в КВ полосах аммиака выбор аналитической пары линий для одновременной регистрации $\mathrm{NH}_{3}$ и $\mathrm{CO}_{2}$ в выдыхаемом воздухе с помощью одного ПДЛ очень ограничен. Наиболее подходящим для этих целей является взаимное расположение линий, образующееся при перекрытии КВ полос этих молекул вблизи $10.3 \mu \mathrm{m}$. Вблизи линии поглощения аммиака $s Q(3.3)$ с центром $967.346 \mathrm{~cm}^{-1}$ и интенсивностью $5.45 \cdot 10^{-19} \mathrm{~cm}^{-1} / \mathrm{mol} \cdot \mathrm{cm}^{-2}$, принадлежащей полосе $0100 a-0000 s$, расположены линии $R(8)$ и $R(10)$ полосы 00011-10001 $\mathrm{CO}_{2}$, имеющие интенсивность $\sim 2 \cdot 10^{-23} \mathrm{~cm}^{-1} / \mathrm{mol} \cdot \mathrm{cm}^{-2}$, причем их удаление от линии NH3 не превышает $1.5 \mathrm{~cm}^{-1}$.

Возможность одновременного измерения концентраций $\mathrm{NH}_{3}$ и $\mathrm{CO}_{2}$ в выдыхаемом воздухе была продемонстрирована с помощью диффузионного лазера на основе соединения $\mathrm{PbSnSe}$, работавшего в диапазоне $10.3 \mu \mathrm{m}$ при температурах $40-60$ К. Экспериментальные лазерные спектры пропускания $\mathrm{NH}_{3}$ и $\mathrm{CO}_{2}$ вблизи $967 \mathrm{~cm}^{-1}$ и их производные показаны на рис. 6. При этом была использована регистрация газов при пониженном суммарном давлении, составлявшем $\sim 100$ Torr. Режим генерации лазера подбирался таким образом, чтобы дифференцированные аналитические линии обоих исследуемых веществ регистрировались в одной моде длительностью $\sim 3 \mathrm{~cm}^{-1}$.

\section{2. Специальные методы накачки пдл}

В ряде случаев можно осуществить регистрацию нескольких газов с помощью одного ПДЛ, используя специальные методы токовой накачки лазера, которые позволяют в одном лазерном импульсе получить последовательную генерацию двух плавно перестраивающихся мод, достаточно далеко разнесенных по частоте. В частности, такой режим реализуется при применении протяженных повторяющихся импульсов тока, содержащих скачкообразное увеличение уровня накачки. Последнее приводит к резкому росту температуры и плотности носителей в активной зоне лазерного кристалла. Как следствие, скачкообразно увеличивается частота генерации лазера во второй половине импульса накачки, причем этот скачок частоты может существенно превосходить тот, который происходит при переключении лазера с одной продольной моды на другую при накачке обычным прямоугольным импульсом тока (как правило, от 1 до $5 \mathrm{~cm}^{-1}$ ). Частота генерации мод, излучаемых до и после токового скачка, может отличаться на несколько десятков $\mathrm{cm}^{-1}$. Величина скачка частоты может управляться величиной перепада уровня токовой накачки. Благодаря последовательной плавной перестройке в достаточно далеко разнесенных по частоте модах это позволяет регистрировать в течение одного лазерного импульса резонансное молекулярное поглощение в спектральных областях, отстоящих друг от друга на десятки $\mathrm{cm}^{-1}$. 
Применение предлагаемого подхода существенно расширяет возможности выбора спектральных участков и линий поглощения, перспективных для многокомпонентного анализа с применением всего лишь одного ПДЛ, так как устраняется необходимость близкого соседства аналитических линий.

Возможность применения предлагаемого метода накачки ПДЛ была нами промоделирована и экспериментально продемонстрирована для нескольких многокомпонентных задач биомедицинской диагностики.

\section{Одновременный анализ $\mathrm{NH}_{3}, \mathrm{CO}_{2}$ и $\mathrm{C}_{2} \mathrm{H}_{4}$}

Использование предлагаемого метода управления зонами перестройки частоты ПДЛ позволяет проводить одновременную регистрацию ряда газов, имеющих интенсивные полосы поглощения вблизи $10 \mu \mathrm{m}$. В этой области с точки зрения медико-биологической диагностики интерес может представлять детектирование $\mathrm{NH}_{3}$, $\mathrm{CO}_{2}, \mathrm{C}_{2} \mathrm{H}_{4}$ и других молекул. В этом случае режим генерации ПДЛ необходимо настроить таким образом, чтобы при низких токах накачки в первой половине импульса регистрировались линии поглощения $\mathrm{C}_{2} \mathrm{H}_{4}$, $R(9.7)$ полосы $v_{7}[19]$, и $\mathrm{CO}_{2}$ вблизи $1023.7 \mathrm{~cm}^{-1}$. Скачок

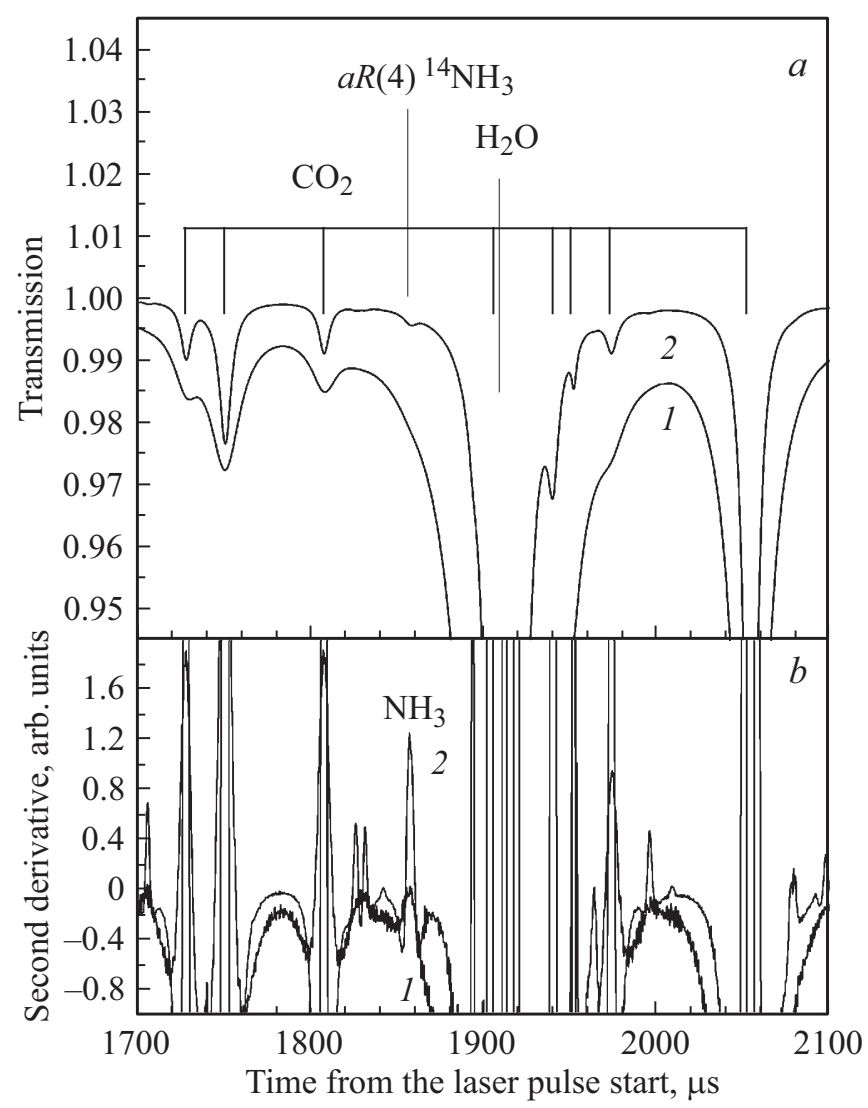

Рис. 6. Спектры пропускания $\mathrm{NH}_{3}$ в выдыхаемом воздухе $(a)$ и их вторые производные $(b)$, детектируемые с помощью ПДЛ вблизи $1065 \mathrm{~cm}^{-1}$. Условия регистрации: концентрации $\mathrm{NH}_{3}-$ $\sim 10 \mu \mathrm{g} / \mathrm{m}^{3} ; \mathrm{CO}_{2}-\sim 3 \% ; \mathrm{H}_{2} \mathrm{O}-\sim 5 \%$; длина оптического пути: $\sim 20 \mathrm{~m}$; суммарное давление 760 (1), 300 Torr (2).

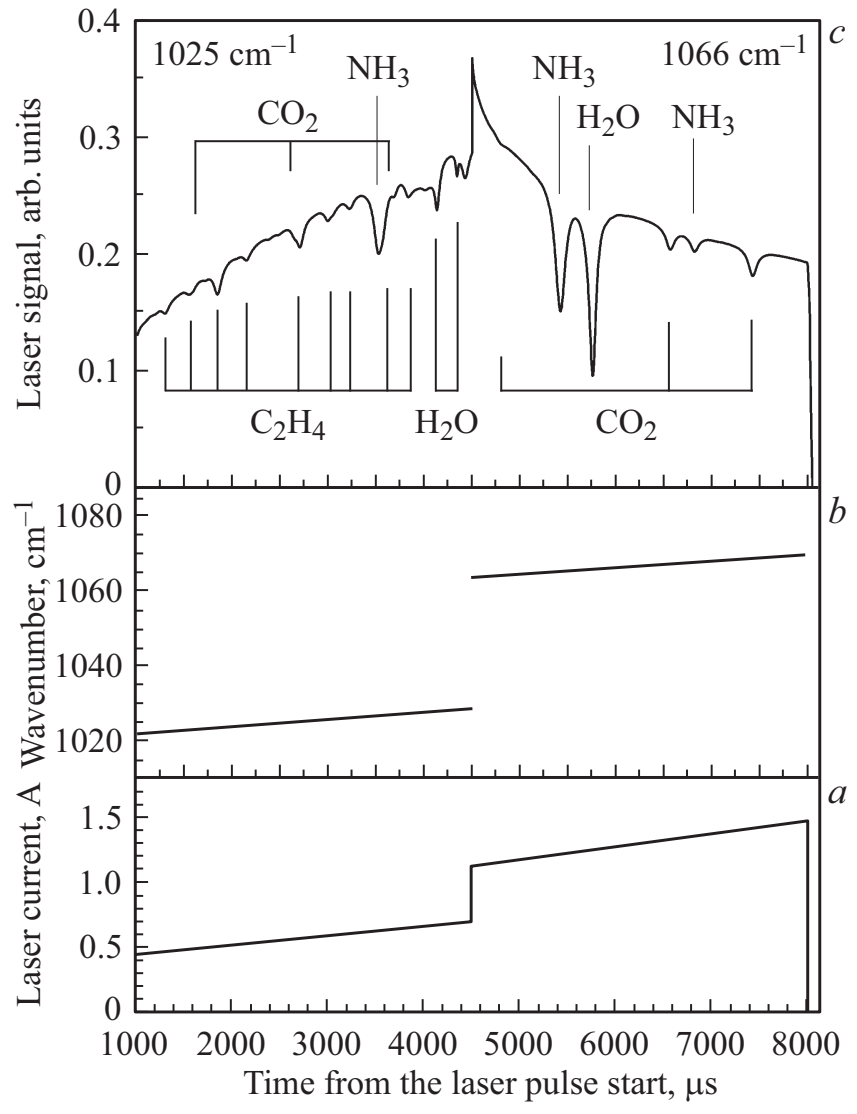

Рис. 7. Использование накачки ПДЛ импульсом тока со скачком амплитуды для многокомпонентного анализа. $a-$ форма импульса накачки. Длительность $\sim 8 \mathrm{~ms}$, стартовая амплитуда $0.32 \mathrm{~A}$, скачок амплитуды $\sim 0.35 \mathrm{~A}$ на $4.5 \mathrm{~ms}$, нарастание $\sim 0.07 \mathrm{~A} / \mathrm{ms}$ (в первой половине), $0.09 \mathrm{~A} / \mathrm{ms}$ (во второй половине). $b$ - модовый состав излучения ПДЛ. $c$ - регистрируемые спектры пропускания смеси выдыхаемого воздуха с этиленом и аммиаком вблизи 1025 и $1066 \mathrm{~cm}^{-1}$. Содержание: $\mathrm{C}_{2} \mathrm{H}_{4} \sim 10 \mathrm{mg} / \mathrm{m}^{3}, \mathrm{NH}_{3} \sim 5 \mathrm{mg} / \mathrm{m}^{3}$. Суммарное давление $\sim 760$ Torr.

тока должен быть подобран так, чтобы во второй половине лазерного импульса детектировался мультиплет аммиака $a R(4)$, располагающийся около $1065.6 \mathrm{~cm}^{-1}$. Таким образом, изменение частоты при перескоке должно составить около $40 \mathrm{~cm}^{-1}$.

Возможность одновременной регистрации $\mathrm{NH}_{3}, \mathrm{CO}_{2}$ и $\mathrm{C}_{2} \mathrm{H}_{4}$ с помощью одного ПДЛ была продемонстрирована с использованием лазера с ДГС на основе соединения $\mathrm{PbSnSe}$. На рис. 7 показан использовавшийся режим накачки ПДЛ, перестроечная кривая и регистрируемые спектры пропускания исследуемых молекул. Отметим, что для достижения требуемых характеристик детектирования потребовалась тщательная селекция лазерных образцов, за счет которой было обеспечено точное попадание в требуемые спектральные области. Для получения желаемой концентрационной чувствительности (на уровне единиц $\mathrm{ppb}$ ) регистрацию этих газов необходимо проводить при пониженном давлении исследуемой 

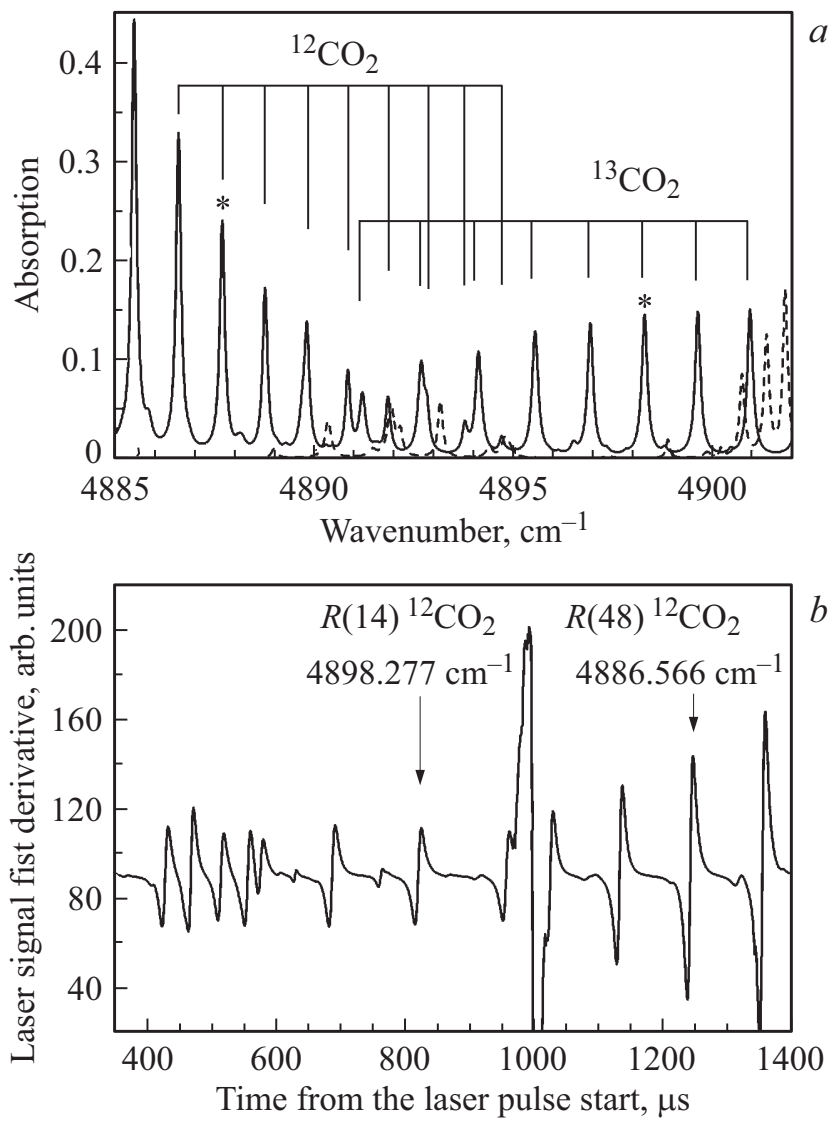

Pис. 8. $a-$ Эмулированный спектр коэффициента поглощения $\mathrm{CO}_{2}$ в районе $2.05 \mu \mathrm{m}$ в атмосферном воздухе с естественным изотопным составом, суммарное давление 760 Torr, парциальное давление $\mathrm{CO}_{2} 22$ Torr, длина оптического пути $5000 \mathrm{~cm}$, использованы спектральные параметры HITRAN-2004. Штриховая линия - спектр поглощения $\mathrm{H}_{2} \mathrm{O} . b-$ Лазерный спектр пропускания $\mathrm{CO}_{2}$ вблизи $2.05 \mu \mathrm{m}$.

газовой смеси, чтобы расширить микроокна прозрачности, которые обусловлены относительно интенсивным поглощением воды и $\mathrm{CO}_{2}$.

\section{Одновременное детектирование $\mathrm{CO}, \mathrm{CO}_{2}$ и $\mathrm{N}_{2} \mathrm{O}$}

Как упоминалось выше, одновременный анализ пар $\mathrm{CO}$ и $\mathrm{CO}_{2}, \mathrm{CO}$ и $\mathrm{N}_{2} \mathrm{O}$ актуален для исследования газообмена в исследованиях по физиологии дыхания и кардиоваскулярной диагностике, проводимых с применением различных нагрузочных тестов, а также для решения проблем анестезиологии.

Дополнительное разнесение частот генерации в начале и конце лазерного импульса позволяет проводить измерение $\mathrm{CO}_{2}$ в диапазоне $2050-2060 \mathrm{~cm}^{-1}$, где расположены линии поглощения более интенсивной полосы 11101-00001. Сила линий в $P$-ветви этой полосы достигает $\sim 2 \cdot 10^{-22} \mathrm{~cm}^{-1} / \mathrm{mol} \cdot \mathrm{cm}^{-2}$. Для детектирования же CO по-прежнему можно использовать наиболее интенсивные линии $P$-ветви полосы 1-0 $\mathrm{CO}$, отстоящие на
$40-50 \mathrm{~cm}^{-1}$ от упомянутых линий $\mathrm{CO}_{2}$ и расположенные в интервале $2100-2110 \mathrm{~cm}^{-1}$.

При одновременном детектировании $\mathrm{CO}$ и $\mathrm{N}_{2} \mathrm{O}$ также удается оптимизировать режим регистрации этих молекул за счет применения ступенчатой накачки. Для анализа СО в этом случае предпочтительно использовать наиболее интенсивные линии $R$-ветви полосы $1-0$, расположенные в интервале $2165-2175 \mathrm{~cm}^{-1}$. А для детектирования $\mathrm{N}_{2} \mathrm{O}$ желательно сместиться в район $2195-2205 \mathrm{~cm}^{-1}$, где наиболее интенсивны линии поглощения этого вещества, принадлежащие $P$-ветви полосы 0001-0000, и на порядок ослабевает поглощение линий $R$-ветви полосы $1-0 \mathrm{CO}$.

Таким образом, применение импульсов накачки со ступенчатым изменением тока позволяет в обоих случаях повысить чувствительность и точность измерений. Кроме того, в случае одновременного детектирования $\mathrm{CO}$ и $\mathrm{N}_{2} \mathrm{O}$ удается снизить концентрацию закиси азота, добавляемой во вдыхаемую смесь, что позволяет увеличить длительность проведения подобных физиологических экспериментов.

\section{Измерение ${ }^{13} \mathrm{CO}_{2} /{ }^{12} \mathrm{CO}_{2}$ вблизи $2 \mu \mathrm{m}$}

Применение импульсов накачки со скачком амплитуды перспективно для измерения изотопического отношения углерода ${ }^{13} \mathrm{C} /{ }^{12} \mathrm{C}$ выдыхаемой $\mathrm{CO}_{2}$ с помощью полупроводниковых лазеров на основе соединений $\mathrm{A}_{3} \mathrm{~B}_{5}$ в диапазоне $2 \mu \mathrm{m}$. В отличие от лазерных структур типа $\mathrm{A}_{4} \mathrm{~B}_{6}$ эти ПДЛ могут работать при температурах выше 200 К. Этот температурный диапазон гораздо более привлекателен для разработки образцов серийных лазерных анализаторов, так как он достижим при использовании термоэлектрических охладителей на основе эффекта Пельтье.

В диапазоне $2 \mu \mathrm{m}$ интерферирует достаточно большое количество так называемых „горячих“ полос и полос, образованных составными колебаниями, обеих молекул. Общее число линий поглощения $\mathrm{CO}_{2}$ в этом диапазоне составляет несколько сотен, но здесь не удается найти рядом расположенные линии поглощения ${ }^{13} \mathrm{CO}_{2}$ и ${ }^{12} \mathrm{CO}_{2}$ с близкой интенсивностью. Однако наблюдается ряд линий, пригодных для проведения изотопического анализа. В частности, при измерении изотопического соотношения ${ }^{13} \mathrm{CO}_{2} /{ }^{12} \mathrm{CO}_{2}$ содержание ${ }^{12} \mathrm{CO}_{2}$ можно контролировать по линии $R(48)$ полосы 20013-00001 с центром на частоте $4886.566 \mathrm{~cm}^{-1}$ и интенсивностью $\sim 2 \cdot 10^{-23} \mathrm{~cm}^{-1} / \mathrm{mol} \cdot \mathrm{cm}^{-2}$. Для определения концентрации ${ }^{13} \mathrm{CO}_{2}$ применима линия $R(14)$ полосы 20012-00001. Она имеет интенсивность $\sim 10^{-23} \mathrm{~cm}^{-1} / \mathrm{mol} \cdot \mathrm{cm}^{-2}$ и расположена вблизи $4898.277 \mathrm{~cm}^{-1}$. При таком расстоянии между аналитическими линиями $\left(\sim 13 \mathrm{~cm}^{-1}\right)$ затруднительно получить одинаковое качество регистрации обеих линий в едином лазерном импульсе, если для накачки используются обычные прямоугольные импульсы. 
Решение проблемы упрощается при применении импульса накачки со ступенчатым увеличением тока, что было продемонстрировано экспериментально с использованием ДГС-лазера на основе соединений GaInAsSb/InAsSbP [20,21]. Такой режим позволяет снизить скорость перестройки частоты лазера в моменты прохождения аналитических линий, более точно управлять диапазоном непрерывной перестройки в используемых модах, применять единую частоту оцифровки спектра и избавляет от необходимости регистрации избыточной спектральной информации. На рисунке 8 показаны эмулированный спектр коэффициента поглощения в диапазоне 4885-4905 $\mathrm{cm}^{-1}$, где наблюдается перекрытие полос ${ }^{12} \mathrm{CO}_{2}$ и ${ }^{13} \mathrm{CO}_{2}$, и сигнал первой производной лазерного спектра пропускания $\mathrm{CO}_{2}$, полученный при использовании ступенчатого импульса накачки. Отметим, что перестройка частоты в лазерах данного типа при нагреве происходит в сторону уменьшения волнового числа. Звездочками и стрелками на рисунке указаны две линии поглощения, наиболее удобные для измерения изотопического отношения ${ }^{13} \mathrm{CO}_{2} /{ }^{12} \mathrm{CO}_{2}$.

\section{4. Заключение}

Таким образом, нами было экспериментально опробовано нескольких различных подходов к многокомпонентному высокочувствительному газовому анализу с использованием методов ДЛС. Они основаны на близком расположении в спектре линий поглощения исследуемых газов и применении специальных методов накачки, позволяющих расширить спектральный диапазон генерации ПДЛ в течение одного импульса. Возможности этих подходов были апробированы при решении задач высокочувствительного анализа состава выдыхаемого воздуха для целей биомедицинской диагностики.

Показано, что одновременный лазерный спектральный анализ пар $\mathrm{CO}$ и $\mathrm{CO}_{2}, \mathrm{CO}$ и $\mathrm{N}_{2} \mathrm{O}$ в районе $4.6 \mu \mathrm{m}$ может быть использован для исследований газообмена в исследованиях по физиологии дыхания и кардиоваскулярной диагностике, проводимых с применением различных нагрузочных тестов, а также для решения проблем анестезиологии и непрерывного мониторинга вентиляционноперфузионного отношения. Одновременный анализ NO и $\mathrm{CO}_{2}$ в районе $5.4 \mu \mathrm{m}$ может быть полезен для контроля маневра дыхания при исследованиях воспалительных процессов в дистальных отделах легких. Одновременное детектирование $\mathrm{NH}_{3}, \mathrm{CO}_{2}$ и $\mathrm{C}_{2} \mathrm{H}_{4}$ вблизи $10.5 \mu \mathrm{m}$ перспективно для исследований общего обмена и основных метаболических циклов. Одновременный анализ ${ }^{13} \mathrm{CO}_{2}$ и ${ }^{12} \mathrm{CO}_{2}$ в районе $2.05 \mu \mathrm{m}$ может быть использован для измерения отношения ${ }^{13} \mathrm{CO}_{2} /{ }^{12} \mathrm{CO}_{2}$ при проведении изотопических дыхательных тестов. Приведены результаты анализа перспективных спектральных диапазонов и взаимного расположения аналитических линий в них. Экспериментально продемонстрирована возможность одновременного детектирования нескольких исследуемых молекул в предложенных спектральных областях, получены лазерные спектры пропускания. Продемонстрирована возможность применения предложенного подхода для анализа микросостава выдыхаемого воздуха.

\section{Финансирование работы}

Работа выполнялась в рамках программы исследований, запланированных в Институте общей физики им. А.М. Прохорова РАН.

\section{Конфликт интересов}

Авторы заявляют, что у них нет конфликта интересов.

\section{Список литературы}

[1] Di Francesco F., Fuoco R., Trivella M.G., Ceccarini A. // Microchemical J. 2005. V. 79. N 1-2. P. 405-410. doi 10.1016/j.microc.2004.10.008

[2] Cao W., Duan Y. // Clinical Chemistry. 2006. V. 52. N 5. P. 800-811. 2006. doi 10.1373/clinchem.2005.063545

[3] Lourenço C., Turner C. // Metabolites. 2014. V. 4. P. 465. doi 10.3390/metabo4020465

[4] Horváth I., Lázár Z., Gyulai N., Kollai M., Losonczy G. // European Respiratory J. 2009. V. 34. N 1. P. 261-275. doi 10.1183/09031936.00142508

[5] Kim K.-H., Jahan S.A., Kabir E. // Trends in Analytical Chemistry. 2012. V. 33. P. 1-8. doi 10.1016/j.trac.2011.09.013

[6] Bingi V.N., Stepanov E.V., Chuchalin A.G., Milyaev V.A., Moskalenko K.L., Shulagin Yu.A., Yangurazova L.R. // Trudy Inst. Obshch. Fiz., Ross. Akad. Nauk. 2005. V. 61. P. 189-210.

[7] Wang Ch., Sahay P. // Sensors. 2009. V. 9. N 10. P. $8230-8262$. doi $10.3390 / \mathrm{s} 91008230$

[8] Hamilton D.J., Orr-Ewing A.J. // Appl. Phys. B - Lasers Opt. 2011. V. 102. P. 879-890. doi 10.1007/s00340-010-4259-4

[9] Stepanov E.V., Milyaev V.A., Selivanov Yu.G. // PhysicsUspekhi.2000. V. 43. P. 417-421. doi 10.1070/PU2000v043n04ABEH000720

[10] Stepanov E.V., Milyaev V.A. // Quantum Electron. 2002. V. 32. N 11. P. 987 doi 10.1070/QE2002v032n11ABEH002333

[11] Stepanov E.V. // Phys Wave Phen. 2007. V. 15. N 3. P. 149-181. doi 10.3103/S1541308X0703003X

[12] Stepanov E.V. // Phys Wave Phen. 2007. V. 15. N 4. P. 241-262. doi 10.3103/S1541308X07040036.

[13] Ventrillard-Courtillot I., Gonthiez T., Clerici C., Romanini D. // J. Biomed. Opt. 2009. V. 14. N 6. 064026. doi 10.1117/1.3269677

[14] Shorter J.H., Nelson D.D., McManus J.B., Zahniser M.S., Sama S.R., Milton D.K. // J. Breath Res. 2011. V. 5. N 3. 037108. doi 10.1088/1752-7155/5/3/037108

[15] Risby T.H., Tittel F.K. // Opt. Eng. 2010. V. 49. N 11. 111123. doi 10.1117/1.3498768

[16] Henderson B., Khodabakhsh A., Metsálá M., Ventrillard I., Schmidt F.M. , Romanini D., Ritchie G.A.D., Hekkert S., Briot R., Risby T., Marczin N., Harren F.J.M., Cristescu S.M. // Appl. Phys. B. 2018. V. 124: 161. https://doi.org/10.1007/s00340-018-7030-x 
[17] Rothman L.S., Jacquemart D., Barbe A., Chris B.D., Birk M., Brown L.R., Carleer M.R., Chac-kerian C., Chance K., Jr., Dana V., Devi V.M., Flaud J.-M., Gamache R.R., Goldman A., Hartmann J.-M., Jucks K.W., Maki A.G., Mandin J.-Y., Massie S.T., Orphal J., Perrin A., Rinsland C.P., Smith M.A.H., Tennyson J., Tolchenov R.N., Toth R.A., Vander A.J., Varanasi P., Wagner G. // J. Quant. Spectrosc. Radiat. Transfer. 2005. V. 96. N 2. P. 139-204. doi 10.1016/j.jqsrt.2004.10.008

[18] Уэст Джс. Физиология дыхания: Основы / Перевод с англ., М: Мир, 1988. 200 c.

[19] Ponurovskii Ya.Ya., Stepanov E.V. // Atmospheric and Oceanic Optics. 2002. V. 15. N 9. P. 766-770.

[20] Yakovlev Yu.P., Baranov A.N., Imenkov A.N., Sherstnev V.V., Stepanov E.V, Ponurovskii Ya.Ya. // Quantum Electron. 1993. V. 23. N 9 P. $726-729$. doi 10.1070/QE1993v023n09ABEH003156

[21] Popov A., Scheumann B., Mucke R., Baranov A., Sherstnev V., Yakovlev Yu., Werle P. // Infrared Phys. Techn. 1996. V. 37. N 1. P. 117-121. 